\title{
A NEW CHART TO IMPROVE THE EFFICIENCY OF GLAUCOMA DETECTION BY OCULOKINETIC PERIMETRY
}

\author{
R. J. STIRLING, J. D. A. MacLEOD and S. A. VERNON \\ Nottingham
}

\begin{abstract}
SUMMARY
Oculokinetic perimetry (OKP) has been proposed as a method to screen for glaucomatous visual field defects but its use is limited by a high false positive rate. The standard hand-held screening OKP chart uses 26 points. The spiral arrangement of numbers with this chart allows loci to be tested close to fixation but may contribute to the high false positive rate. We have therefore designed and assessed a simplified OKP chart with 22 numbers arranged circumferentially around a central target. The positions of the numbers are such that points between $12^{\circ}$ and $16^{\circ}$ from fixation are tested in all quadrants. Loci closer to fixation are not tested. Fifty eyes from 50 patients with glaucoma and 50 eyes from 50 patients with normal fields were tested with both charts. Of the patients with glaucoma $88 \%$ gave a positive result with the 26 point chart and $90 \%$ gave a positive result with the 22 point chart. The false positive rate of OKP in patients without field loss was $12 \%$ with the 26-point chart and 0 with the 22-point chart $(0.02>p>0.01)$. We therefore propose a modification of the existing OKP chart which reduces the false positive rate in glaucoma detection.
\end{abstract}

Conventional perimetry presents peripheral stimuli to an eye which is fixing on a central target. Oculokinetic perimetry (OKP) utilises eye movement to project a stimulus onto different parts of the retina.' The patient follows an ordered pathway of numbers around a central dot and is asked whether he or she can perceive the central stimulus while fixing on each of the peripheral numbers in turn. Consistently missed numbers are recorded on a test card. The card is simply inverted to allow analysis of any field loss. OKP has been proposed as a method of screening for glaucomatous visual field defects. ${ }^{1 .+}$ Although OKP can produce results comparable to more standard methods of perimetry $^{2}$ there has been a false positive rate of $9 \%$ reported with the hand-held screener. ${ }^{+}$If this chart were used in clinical practice it would result in the referral of many patients without disease to the hospital eye services.

Correspondence to: Mr R. J. Stirling. Department of Ophthalmology. University Hospital. Nottingham NG7 2UH. UK.
A previous study has shown that the positions of numbers on the standard OKP chart may not be optimal and suggested an alternative chart of 22 numbers arranged circumferentially ${ }^{5}$ (Fig. 1). We have compared the standard OKP glaucoma screening chart of 26 numbers arranged in a spiral designed by Damato (Fig. 2) with the new chart to determine whether the efficiency of OKP can be improved.

\section{MATERIALS AND METHODS}

Two groups of patients attending a hospital glaucoma clinic were studied, one group with documented glaucomatous field loss and one control group of ocular hypertensives without field loss.

Patients in the glaucoma group fulfilled the following criteria: (1) Unequivocal glaucomatous field loss documented by Humphrey (24-2 statpac program), Friedmann or Goldmann perimetry within the previous year; (2) intraocular pressure greater than $21 \mathrm{mmHg}$ before treatment; (3) glaucomatous excavation of the optic disc on biomicroscopy: (4) best corrected visual acuity of $6 / 9$ or better; (5) absence of any other ocular disease.

Patients in the control group had to have: (1) Normal fields as documented by Humphrey (24-2 statpac program), Friedmann or Goldmann perimetry within the previous 3 months; (2) intraocular pressure consistently less than $30 \mathrm{mmHg}$ on no treatment; (3) normal appcarance of the optic discs on biomicroscopy; (4) best corrected visual acuity of 6/9 or better; (5) absence of any other ocular disease.

Both OKP charts were tested in each patient on the same day. The first chart to be presented was randomised. Only one eye per patient was tested. In the glaucoma group this was usually the side with the more advanced field loss. An experienced clinician assessed the Humphrey, Friedmann or Goldmann field in the glaucoma group and recorded whether there was an unequivocal relative or absolute defect in each quadrant.

The procedure was explained to the patient and he or she was then allowed to complete the test in the presence 


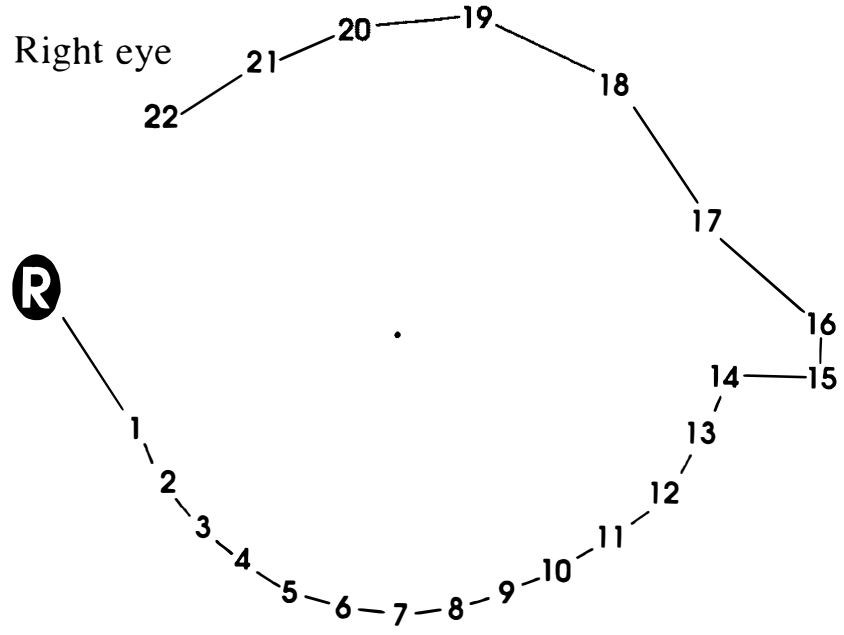

Fig. 1. Modified OKP chart of 22 numbers.

of the examiner but without interruption. If additional help was needed this was recorded. Frail patients or those who had difficulty with standard perimetry were not invited to participate. The opposite eye was occluded by means of a small card attached to the side arm of the chart. This side arm also served to maintain the correct working distance of $40 \mathrm{~cm}$.

A central $1.5 \mathrm{~mm}$ diameter black test stimulus was used with each chart. Fixation and the ability to perceive a positive were checked by asking the patient to look at the letter $\mathrm{R}$ or $\mathrm{L}$ on the chart (depending on which eye was being tested). Fixation on this letter projects the central dot onto the blind spot and only those patients who did not perceive the central dot at this location were asked to proceed with the test. The subjects were then asked to look at each of the numbers in turn, spending approximately 1 second on each, and to report if they could perceive the central dot in their peripheral vision whilst maintaining fixation on the number. The test was repeated and only consistently missed numbers were recorded. The test was positive if the central stimulus was not perceived at one or more numbers.

If available, the patient's own reading glasses were used (bifocal glasses produce 'jump' effects between the near and distance portions of the lens and these were replaced by the spherical equivalent of the reading addition placed in a trial frame). Good lighting conditions from overhead fluorescent units were ensured but not formally standardised for the test.

Statistics were performed using chi-squared analysis of the specificity of the two charts and of the degree of correlation with conventional perimetry. Student's $t$-test was used in analysis of the time taken to perform OKP.

Table I. Type of perimeter used to classify patients into the glaucoma or control group

\begin{tabular}{lcc}
\hline Type of perimeter & Glaucoma group & Control group \\
\hline Friedmann Mk. $24-2$ & 31 & 37 \\
Humphrey 24-2 & 16 & 12 \\
Goldmann & 3 & 1 \\
\hline
\end{tabular}

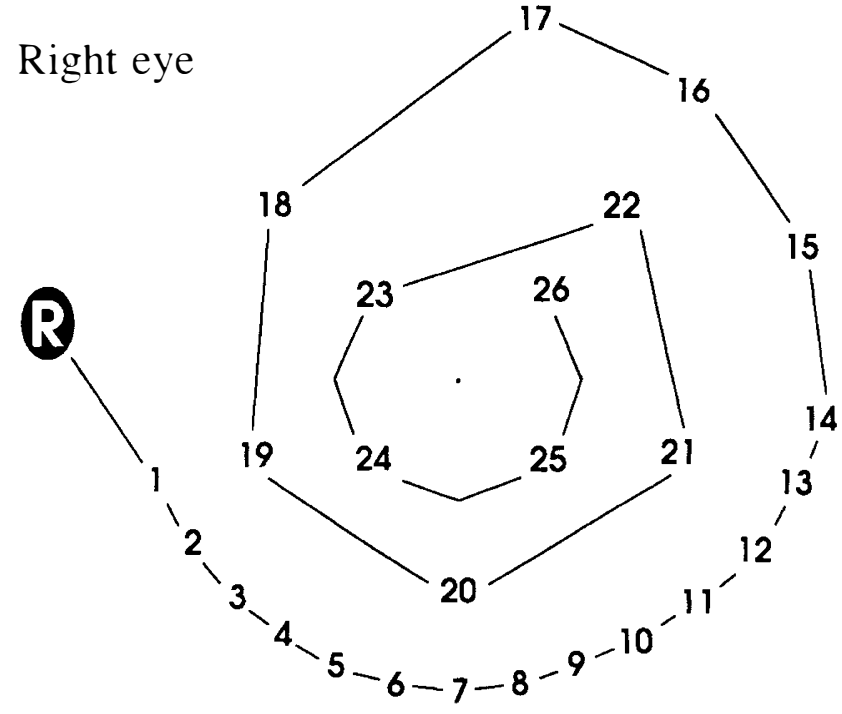

Fig. 2. Original OKP chart of 26 numbers.

\section{RESULTS}

Fifty eyes from 50 patients with glaucoma and 50 eyes from 50 controls with normal fields were tested. Table I shows the type of perimeter used initially to define the presence and extent of any field loss. The age range in the control group was 39-82 years with a mean of 62.9 years (SD 9.4 years). The age range in the glaucoma group was 41-89 years with a mean of 66.7 years (SD 10.7 years).

Forty-five patients in the glaucoma group gave a positive result with the 26-point chart and 5 a false negative response. With the 22-point chart, again 45 patients in the glaucoma group gave a positive response (not all the same patients). Of the 5 false negative responses to the 22-point chart, 3 had also given a negative response to the 26-point chart. The extent of the defects in the glaucoma group as detected by standard perimetry were distributed as shown in Table II.

In patients with glaucoma who gave a positive response to OKP the correlation between the extent of defect assessed by standard perimetry and OKP was determined using three grades:

Grade 1: Identical quadrants detected by OKP and conventional perimetry.

Grade 2: OKP loss correlated with that detected by conventional perimetry in one or more quadrants but there were other quadrants where there was a positive result to OKP but no field loss detected by conventional perimetry or vice versa.

Grade 3: Loss detected by OKP but not in corresponding quadrants to those detected by standard perimetry.

Table II. Distribution of field defects in the glaucoma group

No. of quadrants Relative defects only Relative and absolute defects involved in field loss present (no. of eyes) present (no. of eyes)

\begin{tabular}{lrr}
\hline 1 & 5 & - \\
2 & 7 & 19 \\
3 & 1 & 7 \\
4 & 2 & 9 \\
Total & 15 & 35 \\
\hline
\end{tabular}




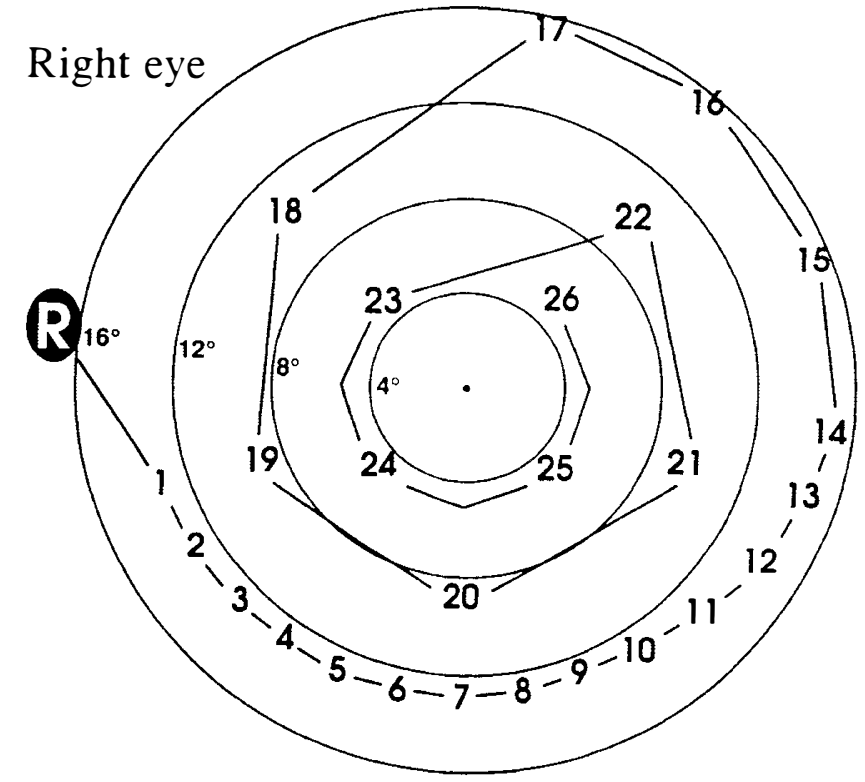

Fig. 3. The 26-point chart tests loci between $5^{\circ}$ and $15^{\circ}$ from fixation.

Exactly corresponding quadrants (grade 1) were identified by OKP in 69\% using the 22-point chart and $49 \%$ with the 26-point chart. A useful level of correlation (grade 2) was achieved in a further $31 \%$ of patients with the 22 -point chart and $49 \%$ with the 26-point chart. One patient in the glaucoma group had a positive result with the 26-point OKP chart but the missed numbers were not in a corresponding quadrant to that detected by the Friedmann mark 2. This OKP result was therefore classified as a false negative in this study.

The 22 point chart has two numbers ( 15 and 16 ) which are displaced peripherally to test for a nasal step. Fourteen patients in the glaucoma group had a nasal step defined by standard perimetry. This was correctly identified by a positive result at either number 15 or 16 (depending whether the defect was above or below the horizontal meridian respectively) in 6 patients.

Six patients in the control group gave a false positive result with the 26-point OKP chart. The numbers that were positive were all in the inferior outer ring of the spiral (i.e numbers $1-15)$. There were no false positive results recorded with the 22-point chart $(0.02>p>0.01)$.

Six patients with normal fields and 21 patients with glaucoma required additional help with one or other charts after the initial explanation.

The average time taken to complete the first run of the 22-point test was 38 seconds in the control group and 1 minute 18 seconds in the glaucoma group. The 26-point test took longer to complete, with a mean of 55 seconds in the control group and 1 minute 38 seconds in the glaucoma group. The 22-point chart was completed quicker than the 26-point chart in both the control group $(p<0.001)$ and the glaucoma group $(p=0.064)$.

\section{DISCUSSION}

The spiral arrangement of the original 26-point chart

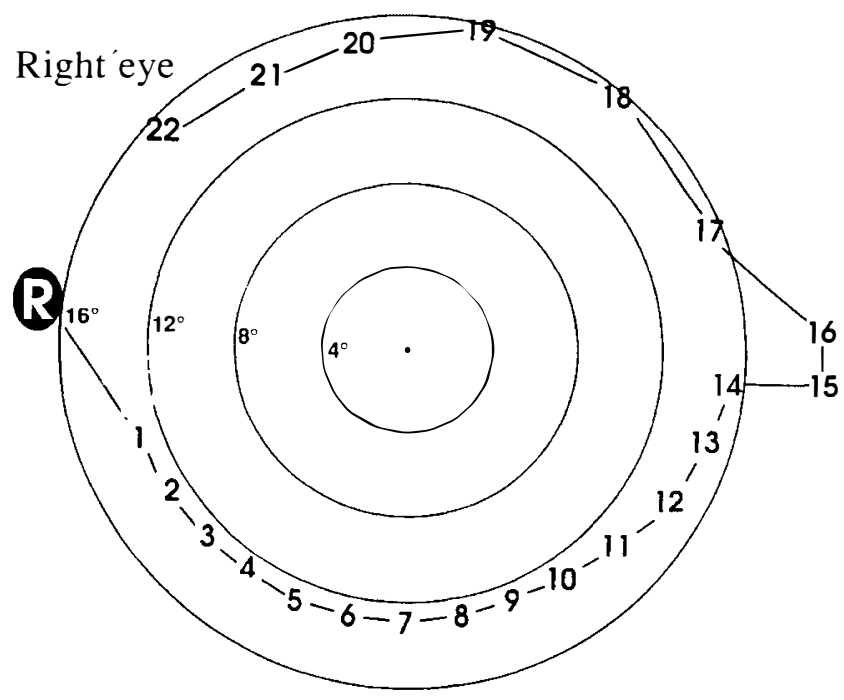

Fig. 4. The 22-point chart does not test loci less than $12^{\circ}$ from fixation. Two numbers are further displaced peripherally to test for a nasal step.

allows points close to fixation to be tested. Numbers greater than 17 test loci within $12^{\circ}$ and four loci (numbers 23. 24, 25 and 26) test points at $5^{\circ}$ of eccentricity in each quadrant (Fig. 3).

Testing points within $12^{\circ}$ from fixation with the 26point chart did not pick up any patients who had not already missed one or more peripheral numbers. Indeed 22 patients in the glaucoma group had well-documented field loss within $12^{\circ}$ of fixation and of these only 9 gave a positive result to any OKP number greater than 17. Because of the doubtful value of the central test points the new 22point chart is designed so that loci within $12^{\circ}$ from fixation are not tested (Fig. 4).

The spiral arrangement of numbers in the original 26point chart brings the locus testing the inferotemporal quadrant (number 18 ) to $10^{\circ}$ from fixation. It has previously been shown that the most sensitive position for glaucoma detection by OKP in this quadrant is at $12.5^{\circ}$ of eccentricity. ${ }^{3}$ The new 22-point chart therefore replaces number 18 with three more peripheral loci (numbers 20 , 21 and 22) which continue the arc towards the blind spot. This new arrangement of numbers tests points between $12^{\circ}$ and $16^{\circ}$ in each quadrant. In addition, two numbers are further displaced peripherally to test for a nasal step. These two points detected only $43 \%$ of nasal steps in this series. The original 26-point chart and the new 22-point OKP chart gave a positive result in $88 \%$ and $90 \%$ respectively of patients with glaucomatous field loss. This rate of sensitivity is comparable to earlier studies which compared OKP to suprathreshold static perimetry. ${ }^{2-4}$ When the Humphrey visual field analyser alone is used to detect patients with field loss for recruitment into a study the sensitivity of OKP drops to $60.5 \% .^{6}$ This lower sensitivity may be due to the undoubted benefits of automated threshold perimeters in the detection of early field loss which remains undetected by OKP. The mean defect at points required to give a positive OKP response has been calculated to be 20.8 decibels below age-matched normals. ${ }^{6}$ 


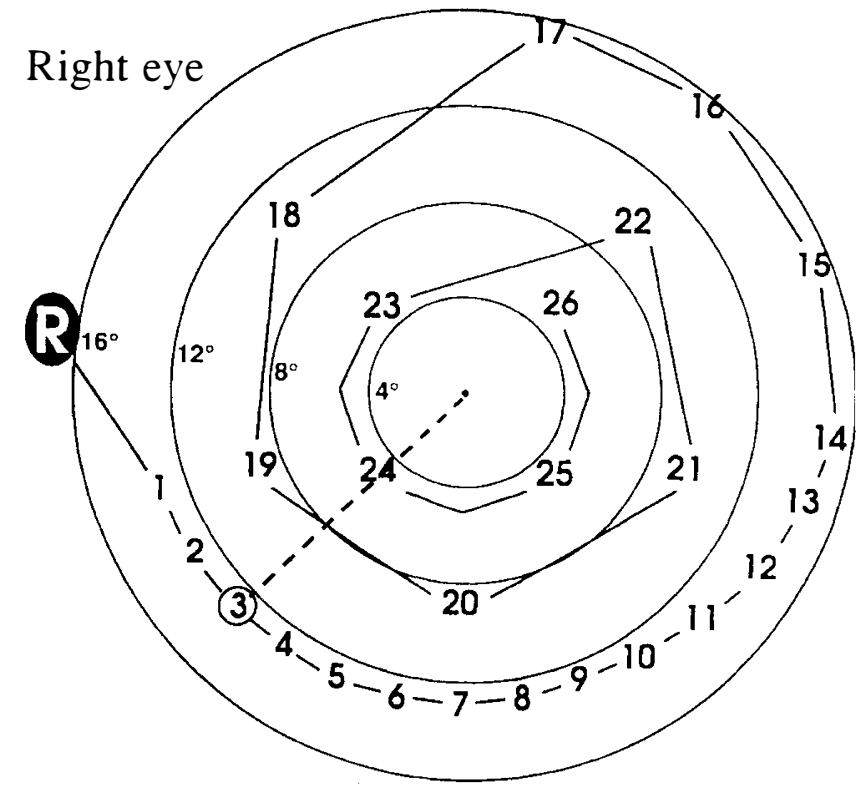

Fig. 5. When testing numbers in the outer ring of the spiral it is possible that confusion may arise from other numbers or line's that are interposed between the peripheral number and the central dot.

The corrected pattern standard deviation (CPSD) is an index of the true deviation of the measured field from the normal hill of vision for the patient's age after correcting for intratest variability (statpac 2 analysis). ${ }^{7}$ At a CPSD of $>4$ OKP has a sensitivity of only $66 \%$ in the detection of glaucomatous defects. With more advanced defects (CPSD of $>6$ ), however, the sensitivity of OKP increases to $92 \% .{ }^{5}$ Our study used the Humphrey visual field analyser in only a minority of patients and tended to select the eye with more advanced field loss for testing. Our aim was, however, not to assess the sensitivity of OKP in the detection of early field loss but to compare two designs of OKP chart.

In spite of fewer test points and only testing loci greater than $12^{\circ}$ from fixation the 22-point chart achieved an equivalent sensitivity to the original 26-point in the detection of glaucomatous defects. There was a greater degree of correlation between the quadrants affected by field loss as detected by standard perimetry and OKP with the 22point chart $(0.10>p>0.05)$ and it may be that simplification of the charts design results in more accurate localisation of field defects. In this series there were no false positives with the 22-point test, whereas there was a $12 \%$ false positive rate with the 26-point chart $(0.02>p>0.01)$.

It would therefore appear that although the spiral arrangement of points with the original chart allows points to be tested close to fixation. where loss is occasionally known to occur in early glaucoma. ${ }^{x .9}$ it may confuse patients and result in a high false positive rate. This confusion may result from the effect of the numbers nearer fixation being interposed between peripheral numbers and the central dot. For example, when the subject looks at point 3 testing the superotemporal field he or she has to 'look through' and ignore number 24 and the line joining numbers 19 and 20 when trying to perceive the central dot
(Fig. 5). This hypothesis would be supported by the fact that all false positive responses with the 26-point chart were to numbers in the outer arc of the spiral (numbers 1-15) where at least two lines or numbers are interposed between the test number and the central dot.

The test procedure was generaly well understood in the control group, with $90 \%$ able to complete the test after the initial explanation without additional help or encouragement. Only $58 \%$ of patients with glaucomatous field loss were able to achieve this. This discrepancy may be because patients with field loss are unwilling to deny the existence of the central dot they have already seen printed on the test chart and therefore tend to lose fixation in an attempt to perceive it. One must be sure that the patient fully understands the test and is confident with it before unsupervised use. The 22-point chart was completed quicker than the 26-point chart in both groups. The glaucoma group took longer with both the 22-point and 26point charts than the patients without field loss.

This study has confirmed that in cooperative patients OKP can achieve a high sensitivity in the detection of well-established glaucomatous defects. The sensitivity of OKP falls when testing defects that are closer to agematched normals. ${ }^{5} \mathrm{OKP}$ is by its very nature a subjective test and it is not appropriate for detecting the earliest glaucomatous field defects. OKP may, however, have a place where facilities for standard perimetry are limited, such as in developing countries where glaucoma tends to present later with dense field loss.

The 22-point chart would appear to be as sensitive as the 26-point chart in a population of hospital out-patients with glaucomatous field defects. As there was a significantly lower false positive rate in our study it may have a greater efficiency when used for screening. A community-based study is now required to confirm this.

Key words: Glaucoma. Oculokinetic perimetry.

\section{REFERENCES}

1. Damato B. Oculokinetic perimetry: a simple visual field test for use in the community. Br J Ophthalmol 1985;69:927-31.

2. Alvarez E, Damato B, Jay J, McClure E. Comparative evaluation of oculokinetic perimetry and conventional perimetry in glaucoma. Br J Ophthalmol 1988;72:258-62.

3. Damato BE. Ahmed J, Allan D. McClure E, Jay JL. The detection of glaucomatous visual field defects by oculokinetic perimetry: which points are best for screening? Eye 1989:3:727-31

4. Damato B, Chyla J. McClure E, Jay J, Allan D. A hand held OKP chart for the screening of glaucoma: preliminary evaluation. Eye 1990);4:632-7.

5. Vernon SA. Quigley HA. A comparison of the OKP visual field screening test with the Humphrey field analyser. Eye 1992;6:521-4.

6.. Wishart PK. Oculokinetic perimetry compared with Humphrey visual field analysis in the detection of glaucomatous visual field loss. Eye 1993:7:113-21.

7. Haley MJ. The field analyser primer. 2nd ed. San Leandro, California: Allergan Humphrey, 1989.

8. Drance S. The early defects in glaucoma. Invest Ophthalmol 1969;8:84-91.

9. Coughlan M, Friedmann A. The frequency distribution of early visual field defects in glaucoma. Doc Ophthalmol Proc Ser 1981:26:345-9. 